\title{
CNS MALFORMATIONS
}

\section{COWDEN SYNDROME WITH CORTICAL MALFORMATION AND EPILEPSY}

Investigators at the Institute for Research on Mental Retardation and Brain Aging, Troina, and University of Naples, Italy report a case of Cowden syndrome presenting with unilateral perisylvian dysplasia and with drug resistant focal seizures. A 14-year-old girl was born with hemiparesis and at 4 months she presented with seizures. Birth weight, height, and head circumference were above the $90^{\text {th }}$ centile. MRI showed a dysplastic cleft and polymicrogyria in the right Sylvian region. The left cerebellar hemisphere was enlarged with dysplastic and hamartomatous appearance characteristic of LhermitteDuclos disease, part of the Cowden syndrome. At 7 years of age she developed obstructive hydrocephalus with herniation of cerebellar tonsils, relieved by a VP shunt and removal of the cerebellar hamartoma, histologically a cerebellar gangliocytoma. Seizures involving left side were refractory to medications. Intestinal examination showed small duodenal polyps. PTEN sequence analysis showed a de novo missense mutation. (Elia M, Amato C, Bottitta M, et al. An atypical patient with Cowden syndrome and PTEN gene mutation presenting with cortical malformation and focal epilepsy. Brain Dev 2012 Nov;34(10):873-6). (Respond: Dr Marco Carotenuto, Second University of Naples, Napoli, Italy. E-mail: marco.carotenuto@unina2.it).

COMMENT. Cowden (or multiple hamartoma) syndrome, named after the first patient reported (Lloyd KM, Dennis M. Ann Intern Med 1963 Jan;58:136-42), is characterized by macrocephaly, intestinal hamartomatous polyps, benign skin tumors, and dysplastic gangliocytoma of the cerebellum (Lhermitte-Duclos disease). The occurrence of drug resistant epilepsy with Cowden syndrome is explained by an associated cortical dysplasia.

\section{EPIDERMAL NEVUS SYNDROME ASSOCIATED WITH BRAIN MALFORMATIONS AND MEDULLOBLASTOMA}

Researchers at Juntendo University and Tokyo Women's Medical University, Japan; and University of California, San Francisco, Ca, report a male infant with epidermal nevus syndrome associated with brainstem and cerebellar malformations and neonatal medulloblastoma. Macrocephaly and enlarged fourth ventricle were noted on fetal ultrasound. At birth the patient had epidermal nevi, brain malformations including polymicrogyria, dysmorphic and enlarged midbrain tectum, and enlarged cerebellar hemispheres. The patient died after surgical resection of a medulloblastoma, diagnosed on MRI at 51 days of age. At autopsy, the cerebellum had many foci of heterotopia and the brainstem showed multiple anomalies, including enlarged superior colliculi, hypoplastic pyramidal tracts and dysplasia of inferior olivary nuclei. These malformations extend the spectrum of epidermal nevus syndrome. (Okumura A, Lee T, Ikeno $\mathrm{M}$, et al. A severe form of epidermal nevus syndrome associated with brainstem and cerebellar malformations and neonatal medulloblastoma. Brain Dev 2012 Nov;34(10):881-5). (Respond: Dr Akihisa Okumura, Department of Pediatrics, Juntendo University Faculty of Medicine, Tokyo, Japan. E-mail: okumura@juntendo.ac.jp). 